\title{
Numerical Simulation of Diffusion Absorption Refrigerator
}

\author{
Guoyan Zhang ${ }^{1,2}$, Shengyong Liu ${ }^{1,2, a}$, Jie Lu ${ }^{1,2}$,Jiong Wang ${ }^{1,2}$ and Yongtao Ma ${ }^{1,2}$ \\ ${ }^{1}$ Collaborative Innovation Center of Biomass Energy, Henan Agricultural University, Zhengzhou, 450002, China \\ ${ }^{2}$ Key Laboratory of New Materials and Facilities for Rural Renewable Energy, Henan Agricultural University, Zhengzhou, 450002 , \\ China
}

\begin{abstract}
Based on Fluent software, a mathematical model of thermosyphon pump is established and numerical simulation is carried out to study the influence of riser tube length, tube diameter and immersion ratio on liquid lifting capacity and efficiency. The results showed that: the liquid lifting volume increased with the increase of immersion ratio, whereas the lifting efficiency showed a trend of increasing followed by decreasing. The highest lifting efficiency for a $340 \mathrm{~mm}$ long, $6 \mathrm{~mm}$ diameter riser achieved when the immersion ratio is 0.35 . With the increasing of the height in riser, the velocity of the gas phase close to the wall in the thermosyphon pump was higher than the velocity along the radial direction. In order to enhance fluid interchange, corners of the refrigeration box were designed to be arc-shaped with a higher corner speed and lower temperature.
\end{abstract}

\section{Introduction}

Diffusion absorption refrigeration was invented by Swedish engineers Von Platen and Munters in the 1920s [1], Ammonia solution or lithium bromide solution is used as a working fluid pair, and hydrogen or helium is added as a diffusion gas. The refrigerant water or ammonia are both environmentally friendly natural working fluids. Low-grade thermal energy such as solar energy, waste heat can be used to provide cooling capacity, Maintain indoor low temperature. Because the working fluid circulating in the whole system relies on thermal energy to drive the thermosyphon and the working fluid's own weight to form a static pressure difference, it has no moving parts such as compressors and throttle valves. It has simple structure, low noise, energy saving and power saving, eliminating power peak-valley differences, The advantages of wide application range and low operating cost are one of the key technologies to realize the sustainable development of the refrigeration industry ${ }^{[2-4]}$.

Scholars at home and abroad have done certain research on diffusion-absorption refrigeration. $\mathrm{Lu}$ Leiying ${ }^{[5]}$ studied the diffusion absorption refrigeration device with gas-liquid separation and rectification equipment, which can increase the concentration to obtain a larger refrigeration capacity, and determined the riser structure Parameter selection. Wang Shikuan ${ }^{[6]}$ established a thermodynamic model of a diffusionabsorption heat exchanger, and conducted detailed cycle performance analysis to investigate the influence of working fluid flow and concentration on system performance. Wang Qin ${ }^{[7]}$ used R23 / R32 / R134a as the mixed refrigerant to study the mixed refrigerant diffusion absorption refrigeration cycle. The results show that a lower evaporation temperature can be reached, and the intermediate boiling point component R32 can improve the refrigeration performance of the system and help reduce the evaporation temperature. He Wei ${ }^{[8]}$ proposed a new resistance coefficient modified bubble pump model to study the bubble pump of the diffusion-absorption heat transformer. The experimental results have good predictability and have been verified. Benhmidene ${ }^{[9]}$ conducted research on bubble pumps of different pipe diameters and received heat flux. The results showed that the optimal heat flux is related to the pipe diameter and mass flow, and the minimum heat flux required for pumping is related to the pipe diameter. Zohar $^{[10]}$ has studied the performance of three diffusion absorption refrigeration systems, and the results show that the structure that integrates the generator and the bubble pump has the best cooling effect. Ezzine ${ }^{[11]}$ conducted a parameterized analysis of the R124/DMAC mixed working fluid through computer simulation, and the results showed that the system performance and the minimum evaporation temperature depend on the absorber efficiency and driving temperature. J Chen ${ }^{[12]}$ used the waste heat of the rectifier to heat the dilute solution in the absorber and produced a new type of generator with a heat exchanger. Under the same cooling capacity, the refrigeration coefficient is increased by $50 \%$.

Based on the previous research, this paper uses ammonia solution as the working fluid pair and hydrogen as the diffusion gas. Through experimental research, under the optimal input power of the refrigerator, the refrigeration system is analyzed theoretically and numerically through the method of numerical simulation, to conduct simulation research on the temperature field and velocity field in the

\footnotetext{
a Corresponding author: liushy@vip.sina.com
} 
thermosyphon pump and the refrigeration box of the diffusion absorption refrigerator to provide a certain theoretical basis for the refrigeration system.

\section{Numerical simulation of thermosiphon pump}

\subsection{Working principle and structure of thermosyphon pump}

The thermosyphon pump is a device that uses heat energy to transport liquid. It inputs heat through the heating device at the bottom, so that the concentrated solution in the tube absorbs heat and vaporizes to produce a two-phase flow state. The bubble lifts the liquid during the rising process. Thermosyphon pump is the core component of the absorption refrigeration system, and the power of the entire system ${ }^{[13-14]}$, the structure of the thermosyphon pump is shown in Figure 1.

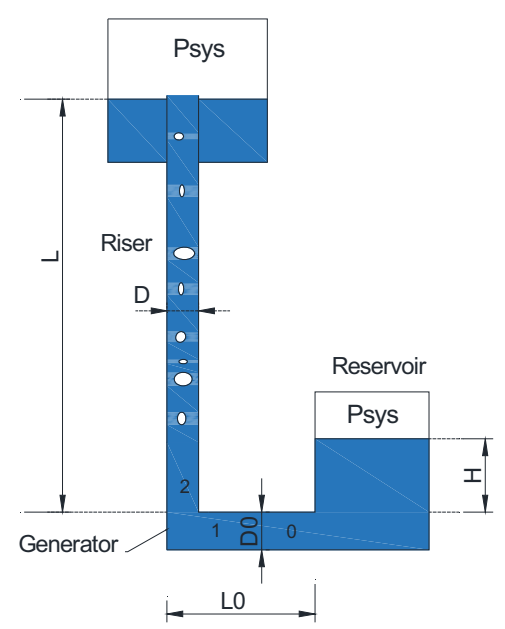

Figure 1. Structure diagram of thermosyphon pump

The inlet of the riser is point 2 , the system pressure is Psys, $\mathrm{D}$ is the inner diameter of the thermosyphon pump riser, $\mathrm{L}$ is the height of the riser, $\mathrm{H}$ is the dynamic pressure head, which is the height of the liquid level of the reservoir, and $\mathrm{H} / \mathrm{L}$ is the immersion ratio, Ignore the frictional resistance loss at the entrance of the riser and the fluid momentum change in the pipe section.

\subsection{Establishment of thermosyphon pump model}

The experiment uses Fluent to simulate the thermosyphon pump. The working fluid circulation model is based on the conservation of mass and energy. In order to simplify the calculation, the following assumptions are proposed:

(1) The flow in the riser is a stable one-dimensional two-phase flow, and the flow state is a slug flow.

(2) There is no hydrogen in the liquid phase, that is, hydrogen is insoluble in ammonia.
(3) Both the gas phase and the liquid phase are incompressible fluids, and the density of each phase is constant.

(4) The system is in adiabatic state and has no dissipation loss.

(5) The gas phase in the cycle is an ideal gas.

The experimental simulation adopts the riser tube lengths of $300 \mathrm{~mm}$ and $340 \mathrm{~mm}$, and the tube diameters of $5 \mathrm{~mm}$ and $6 \mathrm{~mm}$ respectively. Different immersion ratios can be obtained by adjusting the liquid level $\mathrm{H}$ of the reservoir. Under the condition of immersion ratio of 0.2 , $0.25,0.3,0.35,0.4$, the effects of different immersion ratio, tube length and tube diameter on the performance of thermosyphon pumps were studied. The evaluation of the performance of the thermosyphon pump depends on the efficiency of the thermosyphon pump and the quality of the liquid lift carried by the bubble per unit time, that is, the liquid lift volume $m l$. The lifting efficiency is the work done by the thermosyphon pump to lift the liquid under the unit input power, as shown in formula 1 :

$$
\eta=\frac{m \lg (L-H)}{p}
$$

In the formula: $m l$ liquid lifting capacity, $g / s$

$L-H$ Net lifting height of liquid, $m$

$P$ input power, $W$

$L \quad$ Riser length, $m$

$H$ Liquid level height of reservoir, $m$

\subsection{The influence of pipe diameter on thermosyphon pump}

The optimal input power of the diffusion absorption chiller is $48 \mathrm{~W}$ measured through experiments. Under the condition of $48 \mathrm{~W}$ input power and $340 \mathrm{~mm}$ riser tube length, the influence of the riser tube diameter on the liquid lifting capacity and efficiency of the thermosyphon pump is studied as shown in Figure 2. It can be seen from Figure 2 that with the increase of the immersion ratio, the liquid lifting volume and lifting efficiency basically show an increasing trend. The liquid lifting volume of the thermosyphon pump with a pipe diameter of $6 \mathrm{~mm}$ increases with the increase in the immersion ratio, while the lifting efficiency of the thermosyphon pump first increases and then decreases; The liquid lifting volume and lifting efficiency of a thermosyphon pump with a pipe diameter of $5 \mathrm{~mm}$ increase first and then decrease with the increase of the immersion ratio. It can be seen that the immersion ratio has a great influence on the thermosiphon pump. The larger the immersion ratio, that is, the greater the power head $\mathrm{H}$, the smaller the $L-H$, the lifting height of the thermosiphon pump decreases and the lifting resistance decreases, so the immersion ratio Increase, the amount of liquid lift also increases. The small liquid lift of a thermosyphon pump with a diameter of $5 \mathrm{~mm}$ is due to the fact that the liquid lift is related to the resistance along the pipeline, it can be seen from formula (2), the 
smaller the pipe diameter, the greater the pipe resistance along the way, and the smaller the liquid lift.

$$
h_{f}=\lambda \frac{l}{d} \frac{v^{2}}{2 g}
$$

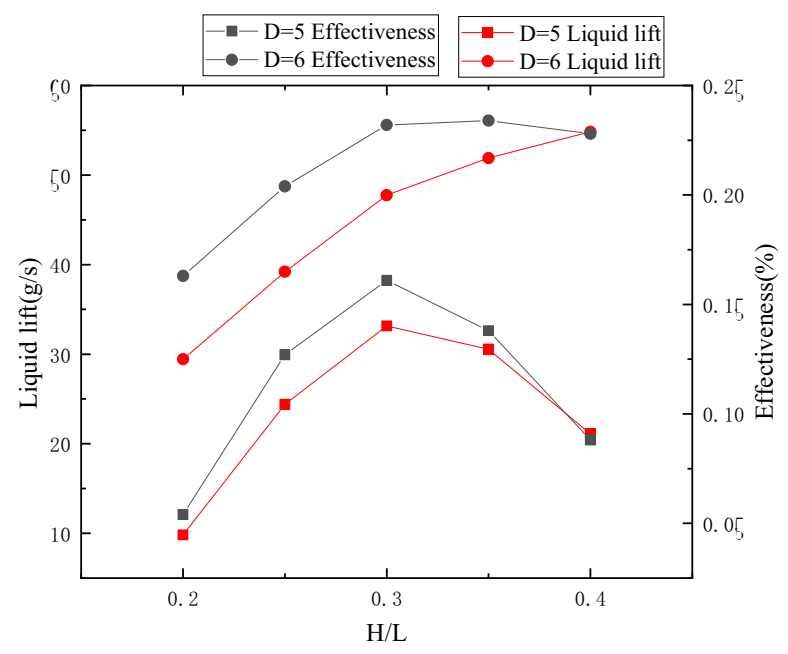

figure 2. The influence of pipe diameter on liquid lifting capacity and efficiency

\subsection{The influence of riser tube length on thermosyphon pump}

Under the condition that the diameter of the thermosiphon pump is $6 \mathrm{~mm}$, the influence of the length of the riser tube of 300 and $340 \mathrm{~mm}$ on the liquid lifting capacity and efficiency of the thermosyphon pump is shown in Figure 3.The liquid lifting capacity increases with the increase of the immersion ratio, the liquid lifting capacity of the riser tube length of $340 \mathrm{~mm}$ is higher than that of the riser tube length of $300 \mathrm{~mm}$;Under certain conditions, it can be seen from formula (1) that the lifting efficiency increases with the increase of the riser tube length, but the riser length cannot be too high, otherwise it will cause the thermosiphon pump to lose its function, reduce the COP of the refrigeration system, and affect the system at the same time The production is running ${ }^{[15]}$. When the immersion ratio is higher than 0.3 , the lifting efficiency decreases. The analysis suggests that the two-phase flow pattern may change from the slug flow to the block flow or annular flow due to the change of the process, resulting in the decrease of the lifting efficiency.

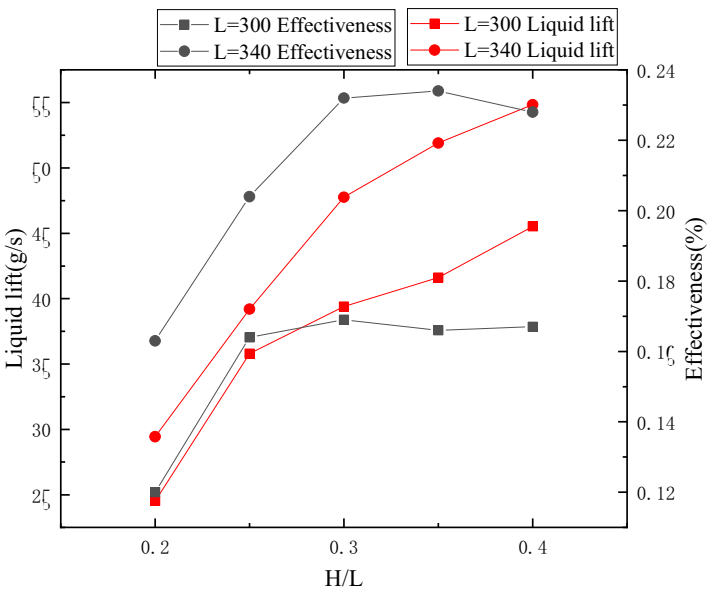

Figure 3. The influence of riser tube length on liquid lifting capacity and efficiency

\subsection{Simulation and analysis of gas phase in thermosyphon pump}

The simulation results of the thermosiphon pump's liquid lifting capacity and efficiency based on the length, diameter and immersion ratio of the riser pipe in the previous section show that the $340 \mathrm{~mm}$ riser has a larger liquid lifting capacity than the $300 \mathrm{~mm}$ pipe length, and the immersion ratio is 0.35 . The lifting efficiency is the highest, so the thermosiphon pump with a pipe diameter of $6 \mathrm{~mm}$, a riser pipe length of $340 \mathrm{~mm}$, and an immersion ratio of 0.35 is selected for further simulation analysis.

After experimental research, the thermosyphon pump is selected when the heating temperature is $438 \mathrm{~K}$. After the system runs stably, the changes in the gas volume fraction cloud diagram at 1, 2, 3, 4, and 5 seconds are shown in Figure 4. After the ammonia solution is heated at the bottom of the generator, the ammonia has a low boiling point, and the ammonia gas is first evaporated. As time increases, the ammonia solution continuously absorbs heat, and the integral number of ammonia gas increases from the bottom to the riser. The small bubbles of vaporized ammonia gas converge into large bubbles, the gas flow gradually increases, and the flow state in the tube is slug. At this time, the efficiency is the highest. The ammonia gas will carry the dilute solution through the solution heat exchanger and send it to the absorber.

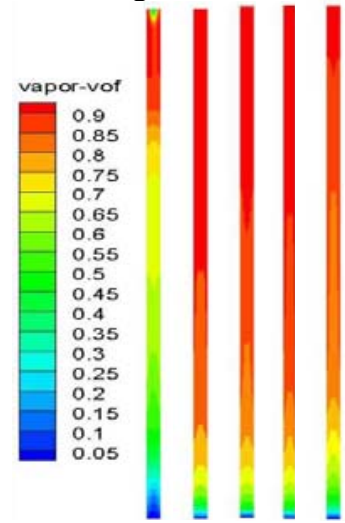

Figure 4. Ammonia integral number cloud chart 
The gas phase velocity vector diagram in the thermosiphon pump is shown in Figure 5. It can be seen from the vector diagram that the gas phase velocity at the lower end of the riser is small. At this time, the thermosiphon pump has just been heated and no bubbles are generated, so the velocity is low. Continue to rise along the riser, you can see that the speed near the wall is higher than the speed along the radius. At this time, the evaporation of the liquid film on the wall accelerates, the amount of air bubbles increases, and the boiling in the thermosyphon pump is strengthened. The wall pushing force is higher than the pushing force along the radial direction, so the wall velocity is higher than the velocity along the radial direction, The liquid lift is the largest, and more dilute solution is lifted out as the bubbles rise.

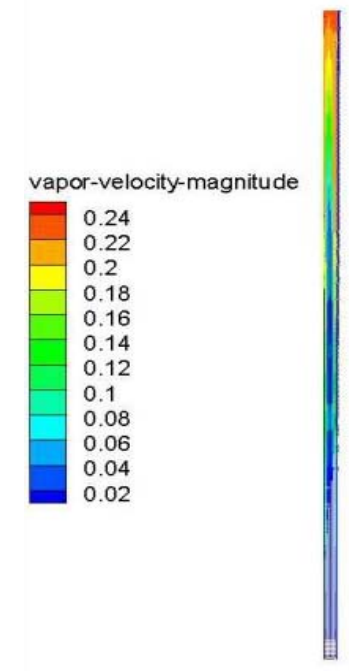

Figure 5. Vapor velocity vector diagram

\section{Analysis of the flow field in the refrigeration box}

In order to understand the temperature and speed distribution in the refrigerating box, Fluent is used to simulate and analyze the flow field in the refrigerating box, study its influencing factors, and propose improvement measures to make the temperature and speed in the refrigerating box meet the design requirements. Therefore, the establishment of the refrigeration box model is shown in Figure 6. The length, width and height of the model are 35,36 , and $44 \mathrm{~cm}$ respectively. A $1.8 \mathrm{~W}$ small fan is installed in the refrigeration box to perform forced convection circulation to make the cold air in the refrigeration box evenly distributed. The simulation analysis is based on the conservation of mass, energy, and momentum. The fan is forced to circulate, and the standard k-e equation and wall function method are used to solve the calculation. To simplify the simulation calculation, the following assumptions are made:

(1) The air in the refrigeration box is an incompressible ideal gas;

(2) The heat transfer of each wall is uniform, and the heat transfer of the fluid in the box is mainly considered;
(3) Ignore the influence of the door opening and closing of the refrigeration box;

(4) The flow field in the refrigeration box is stable and does not change with time;

(5) Ignore the air heat exchange between the inner wall of the refrigeration box and the outer wall.

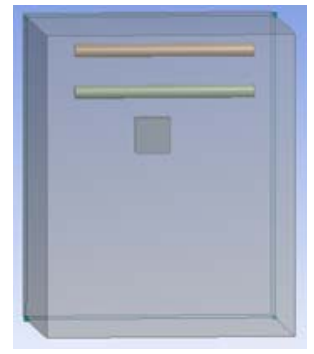

Figure 6. Diffusion absorption refrigeration box model diagram

\subsection{Simulation analysis of temperature and velocity field}

The simulation results of the flow field of the refrigeration box are shown in Figure 7 below. It can be seen from the figure that after the refrigeration box is running stably, the fan distributes the cold generated by the evaporator in the box. It can be seen from Figure 7(b) that the speed in the box is symmetrically distributed along the fan, and the speed at the air outlet is the highest. The cold air flows along the wall of the storage room to the left and right after being blown out by the fan. The speed gradually decreases under the influence of gravity. Figures 7(a) and 7(c) show that the temperature at the corners of the box is higher than that in other areas, and after the cold air is blown out by the fan, the plane area flowing through becomes larger and larger. Interpreted by the flow conservation law ${ }^{[16]}$ formula (3), It can be seen that the flow velocity gradually decreases, and after encountering the wall obstructing the turbulence, the kinetic energy decreases, forming eddy currents at the corners, causing the heat and mass transfer rate to decrease, and the heat at the corners is difficult to be taken away, so the temperature at the corners is relatively high. In order to solve the phenomenon of high temperature at the corners, the corners are designed to be curved to enhance the gas flow and change the direction of the air flow to make the temperature distribution in the box uniform.

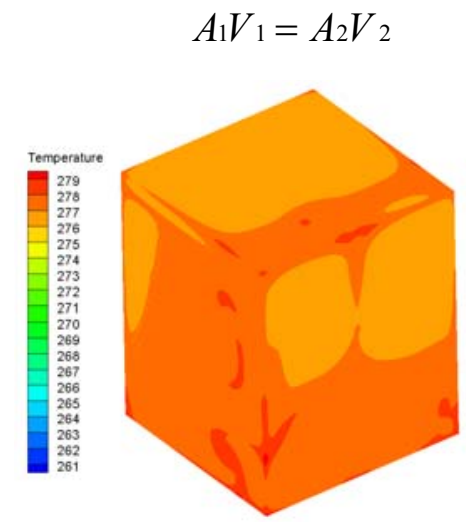

a. Overall temperature chart of the refrigeration box 

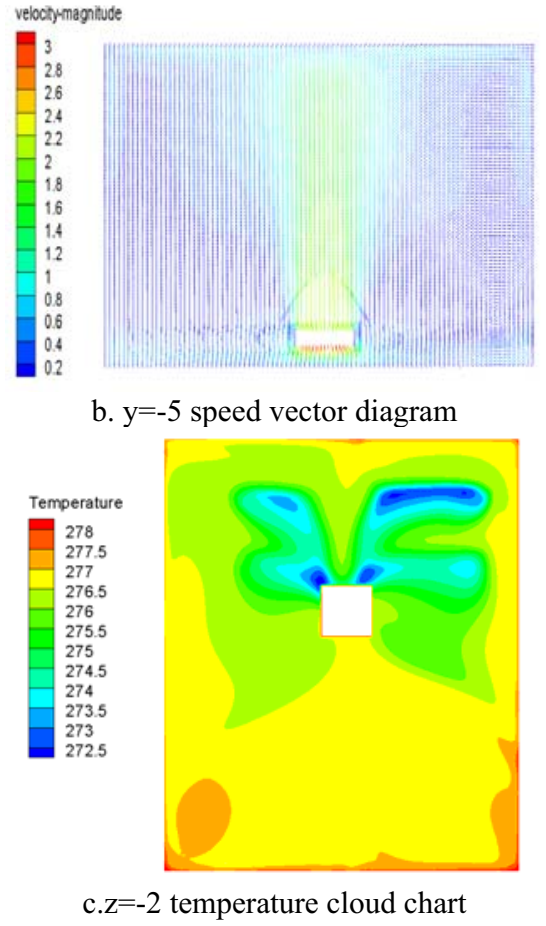

Figure 7. Simulation analysis of the flow field in the refrigeration box

\subsection{Flow field analysis after improved design}

The simulation analysis in the previous section shows that the temperature at the corners of the refrigeration box is relatively high, so the refrigeration box is designed to be chamfered, and the four right angles are designed into an arc as shown in Figure 8. Import the model shown in Figure 8 with the same boundary conditions as in the previous section into Fluent for simulation calculation.

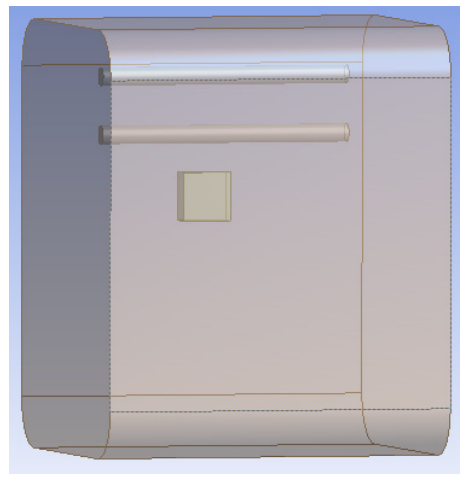

Figure 8. Cooling box model

The simulation result is selected and compared with the same cross section of the refrigeration box before the improved design, as shown in Figure 9. It can be seen from Figure 9(a) that the corner temperature after the refrigeration box is designed into an arc is lower than the corner temperature at a right angle, and the low temperature area in the box is larger than the low temperature area before the improvement. From the speed pattern of $X=-13$ plane in Figure 9(b), The cold air is blown out by the fan and flows to the left and right ends after obstructing the turbulence on the wall. When the corners of the refrigeration box are at right angles, the circulation of cold air is blocked, The air flow speed is reduced, and the heat in the box is not easily taken away; When flowing through the arc-shaped corner, the cold air flows close to the corner, and the area of the low-speed area decreases. The flow heat transfer is enhanced, and the heat at the corner is taken away, so the temperature at the corner is lower than before the improvement.
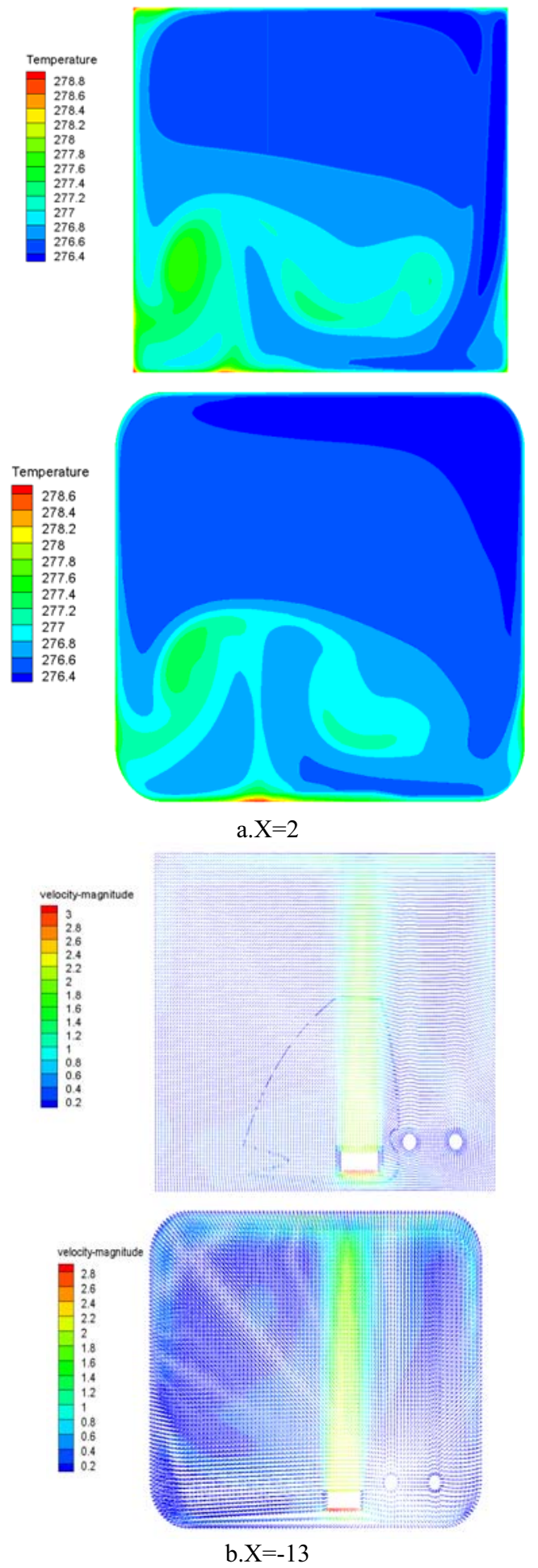

Figure 9. Comparative analysis of the flow field before and after the improvement of the refrigeration box 


\section{4 conclusion}

Energy shortages and environmental issues have been the subject of intense scholarly debate. Diffusion absorption refrigeration has attracted more and more attention of scholars. This paper established a numerical simulation model for thermosyphon pump to study the influence of riser tube length, tube diameter, and immersion ratio on the liquid lifting capacity and efficiency. Based on the model analysis of flow field in the refrigeration box, the following conclusions were drawn:

(1) The immersion ratio plays an important role in the thermosyphon pump. The liquid lift volume increased with the increase of the immersion ratio, and the lift efficiency was first increased and then decreased with the immersion ratio.

(2) The liquid lifting capacity and lifting efficiency of thermosyphon pump with pipe diameter of $6 \mathrm{~mm}$ were higher than pipe diameter of $5 \mathrm{~mm}$. The liquid lifting capacity and lifting efficiency of a riser with a tube length of $340 \mathrm{~mm}$ were greater than those of a $300 \mathrm{~mm}$ riser. The thermosiphon pump with a pipe diameter of $6 \mathrm{~mm}$ and a pipe length of $340 \mathrm{~mm}$ showed the greatest increase in efficiency when the immersion ratio was 0.35 .

(3) When the corners of the refrigeration box were designed to be curved, the circulation of cold air was enhanced, the flow heat exchange was enhanced, and the heat transfer efficiency was improved. The four corner speeds were higher, and the temperature was lower than that before the improved design.

\section{Acknowledgement}

This work was supported by Henan Science and Technology Open Cooperation Project (182106000012).

\section{References}

1. B.V.Platen, C.G.Munters. Refrigerator.US Patent. $(1,685,764,1928)$

2. R.J. Wang, D.P.Liu, X.M. Xue,et al. Design and determination of bubble pump parameters in singlepressure absorption Einstein cycle refrigerator[J].Flu. Mach, (2008:62-65)

3. L.Wang.Principle and Application of Small Absorption Refrigerator[M].C.B.I.P, (2011.1-2)

4. A.Benhmidene, B.Chaouachi, S.Gabsi, et al. Modeling of boiling two-phase flow in the bubble pump of diffusion-absorption refrigeration cycles[J]. Chem. Eng. Commun, 202 (2015)

5. L.Y.Lu, H.Zhang, Z.J.Liu, et al.Performance experiment of a diffusion absorption refrigeration system[J].Cryogenics Supercond, (2007: 79-83)

6. S.K.Wang. Theoretical and experimental research of diffusion-absorption heat transformer[D].(2019)

7. Q.wang, N.Hao, T.F.Sun, Preliminar experimental study on mixed refrigerant diffusion absorption refrigeration system[J].J.Eng. Thermophys, 33(2012, 553-556)

8. W.He.Experimental study on the performance of bubble pump used for diffusion and absorption heat transformer[D].2016

9. A.Benhmidene,B.Chaouachi, S.Gabsi,et al. Modelling of heat flux received by a bubble pump of absorption-diffusion refrigeration cycles[J].Heat Mass Transf, 47, (2011,1341-1347)

10. A.Zohar,M.Jelinek,A.Levy,et al.The influence of the generator and bubble pump configuration on the performance of diffusion absorption refrigeration (DAR) system[J].Int.J.Refrig,31,(2008,962-969)

11. N.B.Ezzine, R.Garma, A.Bellagi.A numerical investigation of a diffusion-absorption refrigeration cycle based on R124-DMAC mixture for solar cooling[J]. Energy,35 (2010,1874-1883)

12. J.Chen, K.J.Kim, K.E.Herold. Performance enhancement of a diffusion-absorption refrigerator [J]. Int.J.Refrig, 19 (1996,208-218)

13. F.M.Zhu, D.P.Liu,L.Y,et al.Research progress of bubble pump in single-pressure absorption refrigeration system[J].J.Refrig,38(2017,65-75)

14. Y.Q.Ping, D.P.Liu, X.M.Xue,et al.Study on steadystate operating characteristics of bubble pump in single-pressure absorption refrigerator[J]. Cryogenics Supercond, 38 (2010, 51-62)

15. M.F.Song,D.P.Liu, W.J.Huang.Development of single pressure absorption refrigeration technology [J].HVAC, (2005,31-35)

16. J.Xie,X.H.Qu,S.Q.Xu.Numerical simulation and verification of gas flow field in cold storage[J].Chs. S.Agri. Eng,(2005,11-16) 